\title{
Locked-Floating-Solid to Locked-Smectic Transition in Colloidal Systems
}

\author{
Jörg Baumgartl, Matthias Brunner, and Clemens Bechinger \\ Physikalisches Institut, Pfaffenwaldring 5, Universität Stuttgart, 70550 Stuttgart, Germany
}

(Received 14 April 2004; published 13 October 2004)

\begin{abstract}
We investigate two-dimensional melting of a colloidal system in the presence of a one-dimensional periodic substrate potential created by two interfering laser beams. We study the commensurability ratio $p=\sqrt{3} a / 2 d=2$ with $a$ the mean particle distance and $d$ the period of the periodic potential. In contrast with the previously investigated case $p=1$, here we observe that melting of the locked-floating solid occurs via a novel locked-smectic phase, predicted by recent theoretical studies.
\end{abstract}

DOI: $10.1103 /$ PhysRevLett.93.168301

PACS numbers: 82.70.Dd, 64.70.Dv

Despite its long history, dating back to the work of Kosterlitz, Thouless, Halperin, Nelson and Young there is continued interest in two-dimensional (2D) freezing and melting (for a review, See [1,2]). While the KosterlitzThouless-Halperin-Nelson-Young theory did not take into account effects of the substrate, is was realized early that this can lead to qualitative changes in the phase behavior (see e.g. [3]).

In their pioneering studies, Chowdhury, Ackerson and Clark experimentally demonstrated the drastic influence of substrate potentials in 2D colloidal model systems using a one-dimensional (1D) periodic laser field [4-6]. When they tuned the periodicity $d$ of the light field (playing the role of the substrate potential) to the first peak in the direct correlation function, at first a modulated liquid (ML) was observed. This phase is characterized by pronounced density modes perpendicular to the potential troughs thus mimicking the geometry of the underlying substrate potential. It was also observed that above a critical laser intensity additional modes along the potential troughs appear which eventually lead to the formation of a 2D crystalline state [locked-floating solid (LFS)] $[4,5]$. Later, it was demonstrated by Monte Carlo simulations, that at even higher laser intensities a remelting to the ML occurs [7]. While this reentrant melting was controversially discussed for some time [8], at first experiments $[9,10]$ but later also theoretical and numerical studies [11-13] confirmed this scenario. This reentrant melting was explained as generically (in systems with short-range interactions) being due to suppression of phonon fluctuations transverse to the substrate troughs, leading to decoupling of the adjacent rows of colloids [9]. Accordingly, if those fluctuations are reduced by increasing the laser intensity, remelting of the crystal occurs.

Recently, it was realized by Radzihovsky, Frey and Nelson that the complexity of the phase diagram largely increases if other commensurability ratios $p=\sqrt{3} a / 2 d$ with $a$ being the mean particle distance and $d$ the period of the 1D periodic potential are considered [11,12]. While the above mentioned scenario is valid for the case $p=1$ (where every potential trough is occupied with particles) for $p=2$ an additional locked-smectic (LSm) state is predicted. In contrast to the LFS, this phase is characterized by short-range correlations between colloids in neighboring troughs and therefore the LSm does not resist shear deformations for displacements along the potential minima. On the other hand, unlike the ML-that breaks no additional spatial symmetries and is invariant under translations by $d$ perpendicular to the 1D periodic potential, the LSm spontaneously breaks this discrete symmetry down to a lower symmetry of translations by $p d$ [12].

Similar to the Kosterlitz-Thouless-Halperin-NelsonYoung theory (KTHNY) scenario, the phase behavior in the presence of 1D substrate potentials as discussed above is generic for systems with short-ranged interactions. For $p=1$, Monte-Carlo studies found reentrant melting for Yukawa-like pair potentials hard disks and even in simulations where quantum fluctuations were taken into account [13-15]. Therefore, the investigation of such phase transitions allow general insights into fundamental properties of 2D systems on patterned substrates.

In this Letter, we present an experimental study on the phase behavior of a 2D colloidal system under the influence of an 1D periodic potential for $p=2$. In contrast to $p=1$ the system has an additional degree of freedom, as the particles can occupy different potential troughs. As a consequence the LFS does not melt directly into the ML but via a novel, intermediate LSm phase which is experimentally observed for the first time. We also find a transition from the LSm to the ML when the pair interaction is reduced. This is in agreement with theoretical studies [12].

We used an aqueous suspension of highly charged polystyrene (PS) spheres with radius $R=1.45 \mu \mathrm{m}$ and an average polydispersity below $4 \%$. The pair-interaction potential between the particles corresponds to a screened Coulomb potential with the Debye screening length $\kappa^{-1} \approx 500 \mathrm{~nm}$, as determined from inversion of the pair correlation function [16]. The sample cell consisted of a fused silica cuvette with $200 \mu \mathrm{m}$ spacing which was connected to a standard closed deionization circuit [9]. The periodic potential was created by interference of two 
linearly polarized beams of a $N d: Y V O_{4}$ laser $(\lambda=$ $532 \mathrm{~nm}$ ). Owing to optical gradient forces, this interference pattern acts as an external potential on the particles [4] with the potential amplitude $V_{0}$ scaling linearly with the laser power [10]. Another laser tweezer was scanned around the central region of our sample to create a boundary box for the system. This allowed us to adjust the particle density to meet $p=2$ [16].

Particle positions were imaged onto a charge-coupled device camera with a acquisition rate of one frame per second and the 2D density distribution $\rho(x, y)$, and the corresponding pair correlation function $\mathrm{g}(\mathrm{x}, \mathrm{y})$ were obtained (Fig. 1). Here $\mathrm{x}$ and $\mathrm{y}$ denote the direction perpendicular and along the laser lines, respectively.

The particle density was kept constant at $\rho=$ $0.026 \mu \mathrm{m}^{-2}$ which is below the value where spontaneous crystallization occurs. Therefore, for $V_{0}=0$, we observed an isotropic 2D liquid (data not shown). The lattice constant $d$ of the 1D potential was adjusted to match the condition $p=2$, i.e. $d=\sqrt{3} a / 4$ with the mean particle distance $a=\sqrt{2 / \sqrt{3} \rho}$. Depending on the substrate strength and the mean particle interaction we observed different phases which are shown in Fig. 1 and which will be discussed in the following.
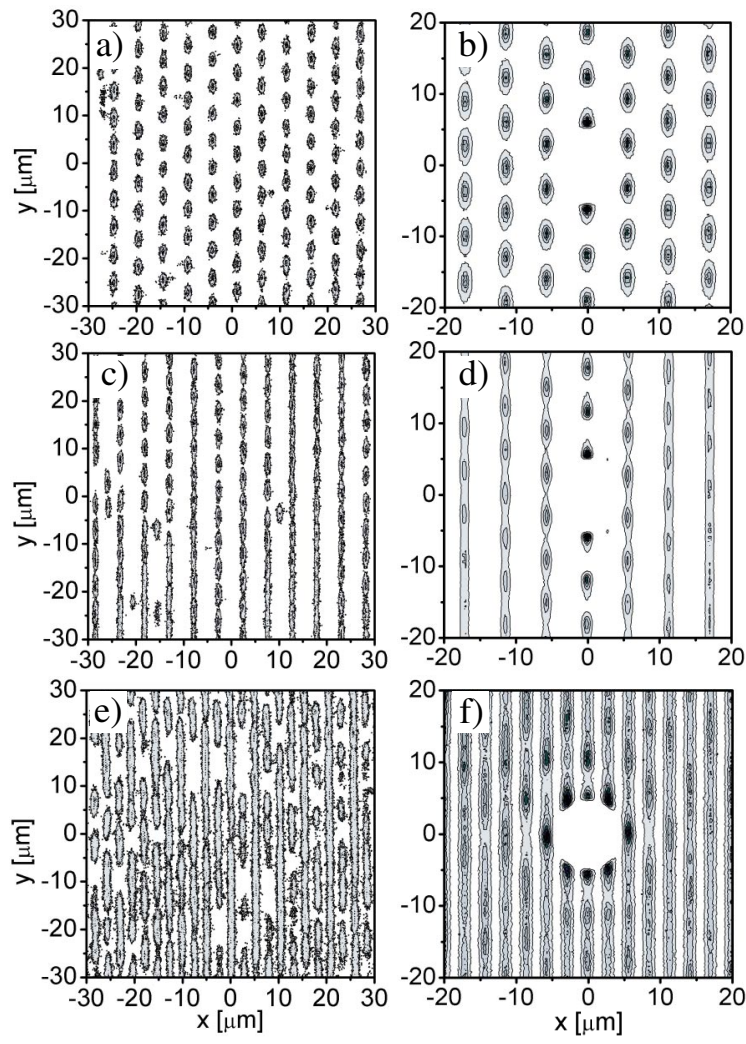

FIG. 1 (color online). Averaged particle density $\rho(\mathrm{x}, \mathrm{y})$ and pair correlation function $\mathrm{g}(\mathrm{x}, \mathrm{y})$ for $(\mathrm{a}, \mathrm{b}) \mathrm{LFS},(\mathrm{c}, \mathrm{d}) \mathrm{LSm}$ and (e, f) ML phase.
With increasing substrate strength $V_{0}$ the particles become more and more localized along the interference fringes due to light forces. Because of the repulsive particle interaction, the system responds at sufficiently high by forming a crystalline (LFS) phase. Figure 1(a) shows the LFS which was obtained for $V_{0}=4.2 k_{B} T$. This situation is very similar to the corresponding phase at $p=1$ where due to the interplay of interparticle and particle-substrate forces a light-induced crystalline state was observed [4,9]. In contrast to $p=1$, however, here on average only every second row is occupied with particles. The corresponding $\mathrm{g}(\mathrm{x}, \mathrm{y})$ in Fig. 1(b) supports the characteristic quasi-long-range order of the LFS along the potential troughs which is responsible for the nonzero shear elasticity.

Upon increasing the laser potential to $V_{0}=7.9 k_{B} T$ the particle density shows a different structure [see Figs. 1(c) and 1(d)]. In contrast to the LFS, now only short-range correlations along the troughs are observed but the density modulation as observed for the LFS perpendicular to the troughs is still preserved. It is important to realize that even at $V_{0}=7.9 k_{B} T$ the potential barriers between the troughs are sufficiently small to be overcome by the particles (as is observed in the experiment). Therefore, the structure presented in Figs. 1(c) and 1(d) is an equilibratet state being identified as LSm phase. Both, the existence of the LSm and, in particular, the transition from the LFS to the LSm upon increasing $V_{0}$ is in agreement with theoretical predictions [11,12].

These predictions also suggest that further increase of the laser potential leads to a transition from the LSm to the modulated liquid. This is caused by a reduction of the particle fluctuations perpendicular to the laser troughs, thus decreasing the effective interaction among particles in neighboring troughs. Accordingly, for short-range pair potentials (as studied here) the interaction energies of the LSm and the ML in the limit of large $V_{0}$ are approximately the same. However, due to entropic contributions the free energy of the ML (where the particles are randomly distributed across all potential troughs) becomes smaller and a transition from the LSm to the ML should occur [12]. This, however, was not observed in our experiments. The absence of this transition is probably due to kinetic reasons because the phase transition from the LSm to the ML requires thermally activated hopping of particles across laser troughs. Due to the high $V_{0}$ values required to induce this transition, this hopping is highly suppressed and one would have to wait an exponentially long-time $\left(\tau \propto \exp \left(V_{0} / k_{B} T\right)\right)$. Therefore, such a transition is not expected to occur on our experimental time scales.

According to Frey et al., a transition from the LSm to the ML should also be observed when reducing the pairinteraction energy [11]. This was experimentally achieved by increasing the salt concentration in the suspension, i.e., decreasing the Debye screening length. 
Figure 1(e) and 1(f) shows the data obtained at a lower screening length (about $\kappa^{-1} \approx 450 \mathrm{~nm}$ ) and a substrate strength of $V_{0}=6.7 k_{B} T$. As can be seen, now all potential troughs are occupied with particles which is a clear signature of the ML. Similar to the case $p=1$ [9] local hexagonal order is observed while correlations along the y-direction rapidly decay [Fig. 1(f)].

A more quantitative description of the phases can be obtained from an analysis of $\mathrm{g}(\mathrm{y})$, i.e., the pair correlation function along the laser lines. Fig. 2(a) shows the corresponding curves for the LFS phase which shows again the rather long-range order along the troughs. According to [12] it is important to take into account leading and subleading quasi-Bragg peaks for the analysis of the paircorrelation function and thus finds

$$
\begin{aligned}
g(y)-1= & \text { const. }+g_{A} \cos \left(G_{y}^{0} y\right) y^{-\eta_{G_{A, y}}} \\
& +g_{B} \cos \left(2 G_{y}^{0} y\right) y^{-\eta_{G_{B, y}}}
\end{aligned}
$$

where $g_{A}$ and $g_{B}$ are amplitudes depending on the strength of the laser potential, and $G_{y}^{0}=2 \pi / a$ and $\eta_{G_{A, y}}, \eta_{G_{B, y}}$ characteristic exponents for the LFS phase. Because of the superposition of the two harmonics with different power law amplitudes the minima of Eq. (1) are much broader than the maxima (a similar behavior was experimentally also observed for $p=1$ [9].) Indeed, Eq. (1) which is plotted as solid line describes the experi-

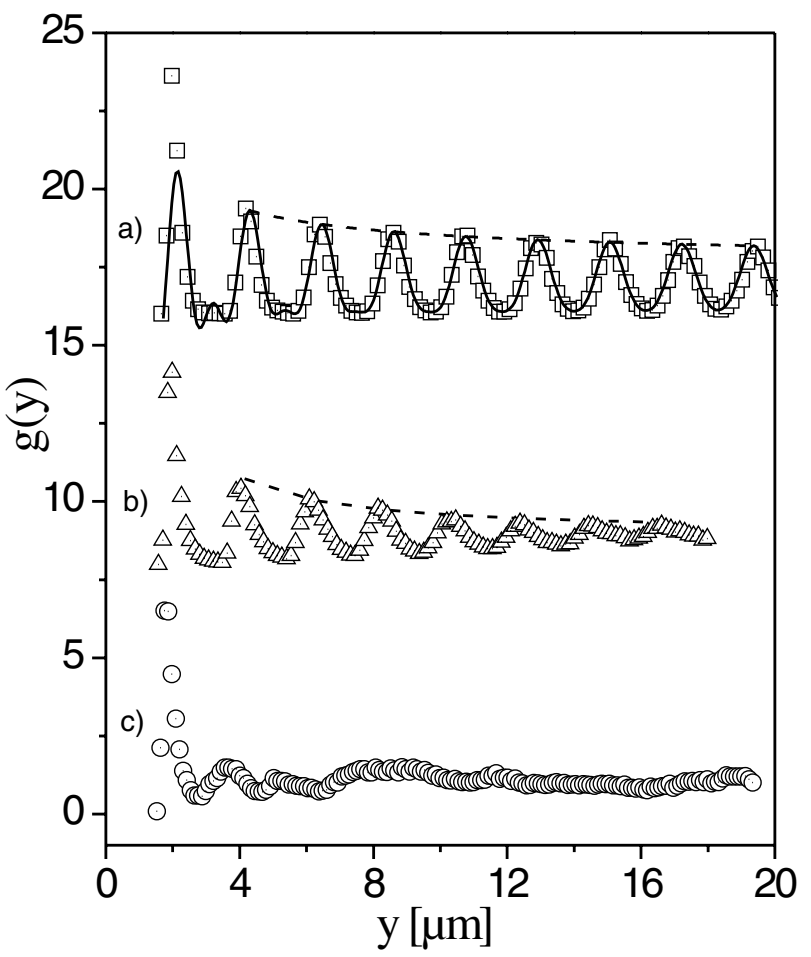

FIG. 2. Pair correlation functions $g(y)$ along the potential troughs for (a) LFS, (b) LSm, and (c) the ML phase. The data are displaced in vertical direction for clarity. The solid and dashed lines are fitting functions as explained in the text. mental data of Fig. 2(a) very well, in particular, the flattened minima are reproduced (we did not try to fit the first peak because it critically depends on the pair potential between the particles which is not taken into account by Eq. (1) [17]). A least mean square fit to the data yields $\eta_{G_{A, v}}=0.27$ and $\eta_{G_{B, v}}=1.19$ which suggests that the observed LFS is close to the melting point [2]. For comparison, we also fitted the peaks in $\mathrm{g}(\mathrm{y})$ with a single algebraic fit (dashed curve). Although it describes the data very well and indeed also supports a crystalline phase, the resulting exponent has to be taken with care [12].

The pair correlation functions of the LSm and the ML phase decay much faster and the envelope at larger distances is best described by a single exponential as seen by the dashed line in Fig. 2(b) (an algebraic fit according to Eq. (1) yields $\eta_{G_{A, y}}>1$ which is beyond the limiting value indicating quasi-long-range order). This clearly demonstrates the short-ranged nature of correlations along the potential troughs in this phase. In the ML phase the correlation function decays even faster and becomes rather noisy beyond distances of $6 \mu \mathrm{m}$. This very fast decay is essentially caused by the largely reduced coupling of particles in adjacent lines.

To understand why the light-induced melting from the LFS to the ML occurs not directly (as for the case $p=1$ ) but via an intermediate LSm phase, one has to recall that at $p=2$ there are two consecutive mechanisms how the system increases its entropy: first, the particles increase their fluctuations along the potential troughs thus forming the LSm. In a second step, the particle positions can distribute equally across all potential lines which then leads to the ML. It should be mentioned, however, that according to Refs. [11,12] there might be also a region in the phase diagram where a direct light-induced transition from the LFS to the ML occurs. While in our experiments we did not observe such a transition this needs further systematic investigations. We speculate at this point, however, that such a direct transition is more likely in systems with long-ranged particle interactions because then fluctuation-induced effects, which are essential for the above phase transitions, are less pronounced.

In addition to static quantitifiers we also analyzed the mean square displacement (MSD) of the particles. Because of the geometry of the substrate potential we separated the particle motion in $\mathrm{y}$ and $\mathrm{x}$ direction and calculated the MSD along $\left(\left\langle y(t)^{2}\right\rangle\right)$ and perpendicular $\left(\left\langle x(t)^{2}\right\rangle\right)$ to the potential troughs [symbols in Figs. 3(a) and 3(b)]. The solid lines correspond to a fit to an analytical expression which allows to obtain the short-time and long-time self-diffusion coefficients $D_{S}$ and $D_{L}$, respectively [18]. While $D_{S}{ }^{y}$ along the troughs is identical for the three phases $\left(D_{S}{ }^{y}=0.04 \mu \mathrm{m}^{2} / \mathrm{s}\right), D_{L}{ }^{y}$ is rather different [Fig. 3(a)]. For the LFS $(\square), \operatorname{LSm}(\Delta)$ and the ML (O) phase we found $D_{L}^{y}=0.001,0.0053$, and 

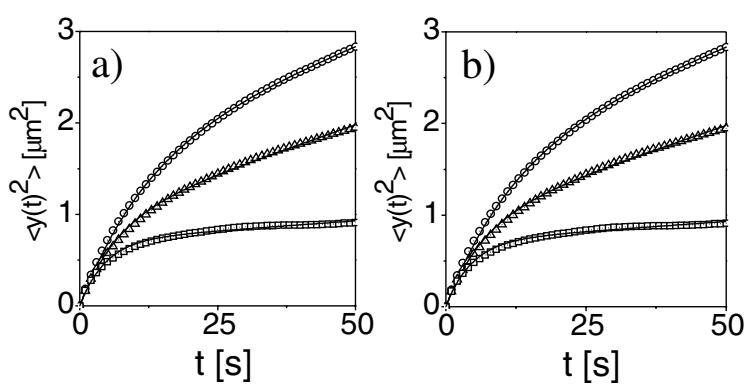

FIG. 3. Mean square displacements of the particle motion (a) along and (b) perpendicular to the interference fringes for LFS $(\square)$, LSm $(\Delta)$ and the ML $(\bigcirc)$ phase. The solid lines correspond to fits which allow to extract the short-time and longtime diffusion coefficients.

$0.006 \mu \mathrm{m}^{2} / \mathrm{s}$. The small value of $D_{L}^{y}$ for the LFS demonstrates the fluctuation-induced strong coupling between particles in this phase. The somewhat smaller diffusion coefficient $D_{L}{ }^{y}$ of the LSm phase compared to the ML reflects the - although weak-but still visible effect of this coupling.

In Fig. 3(b) we plotted $D_{S}{ }^{x}$, i.e., the corresponding diffusion coefficients perpendicular to the potential troughs. Since the particles are strongly confined in this direction by the substrate potential, the values are about 1 order of magnitude smaller. The MSD of the LSm phase is below the data of the LFS because $V_{0}$ was larger for the LSm. In contrast to the LFS and LSm, the ML still shows a significant slope at longer times. This reflects the fact, that in the ML particle hopping across the laser lines is more likely and the particles become less localized within a single potential trough.

Löwen suggested a phenomenological melting criterion based on the ratio of long-time and short-time diffusion coefficients [19]. In the crystalline state this quantity should yield in case of homogeneous $2 \mathrm{D}$ systems $\frac{D_{L}}{D_{S}} \leq$ $0.086 \pm 0.01$. Although it is not clear whether this criterion is also valid in the presence of substrate potentials, it is interesting to calculate the corresponding values for the observed phases. Table I shows the results for the $\mathrm{y}$ and $\mathrm{x}$ directions of particle motion. In agreement with the Löwen-criterion, we find that the values along the potential troughs suggest a crystalline state for the LFS. In contrast, both the LSm and the LM yield values corresponding to a liquid. In the direction perpendicular to the laser potential, the particle densities of all three phases have a periodic, i.e., crystalline behavior due to the periodic intensity modulation in x-direction. As can be seen in the last column of Table I, this is consistent with the above phenomenological melting criterion. Apparently, the dynamical phase criterion is consistent with the phase analysis based on static quantifiers. At this point, however, it is difficult to judge whether this agreement is accidental or whether the criterion can be applied to
TABLE I. Short- and long-time diffusion coefficients $D_{S}$ and $D_{L}$ along (y) and perpendicular (x) to the potential troughs and for different phases as observed in Fig. 1.

\begin{tabular}{lllllll}
\hline \hline & $D_{S}{ }^{y}$ & $D_{L}{ }^{y}$ & $D_{L}{ }^{y} / D_{S}{ }^{y}$ & $D_{S}{ }^{x}$ & $D_{L}{ }^{x}$ & $D_{L}{ }^{x} / D_{S}{ }^{x}$ \\
\hline LFS & 0.04 & 0.001 & 0.025 & 0.04 & 0.000086 & 0.00215 \\
LSm & 0.04 & 0.0053 & 0.13 & 0.04 & 0.00012 & 0.003 \\
ML & 0.04 & 0.006 & 0.15 & 0.04 & 0.0008 & 0.02 \\
\hline \hline
\end{tabular}

systems with substrate potentials. We hope that this work will stimulate further theoretical studies.

In summary, we have investigated the melting behavior of a $2 \mathrm{D}$ colloidal suspension in the presence of a modulated light field for the commensurability ratio $p=2$. In contrast to the case $p=1$, here we observe that the LFS melts via an additional intermediate phase, i.e., a LSm into the ML. Our experimental observations are in qualitative agreement with recent theoretical predictions.

It is a pleasure to acknowledge Leo Radzihovsky for helpful discussions. This work is supported by the DFG (BE1788/3).

[1] K. J. Strandberg, Rev. Mod. Phys. 60, 161 (1988).

[2] D. R. Nelson, Phase Transitions 7, 1 (1983).

[3] V. L. Prokrovsky, Solitons, Vol. Chap.3, p. 71 - 127 (North Holland, Amsterdam, 1986).

[4] A. Chowdhury, B. J. Ackerson, and N. A. Clark, Phys. Rev. Lett. 55, 833 (1985).

[5] B. J. Ackerson and A. H. Chowdhury, Faraday Discuss. Chem. Soc. 83, 309 (1987).

[6] K. Loudiyi and B. J. Ackerson, Physica A (Amsterdam) 184, 1 (1992).

[7] J. Chakrabarti, H. R. Krishnamurthy, A. K. Sood, and S. Sengupta, Phys. Rev. Lett. 75, 2232 (1995).

[8] C. Das, A. K. Sood, and H. R. Krishnamurthy, cond-mat/ 9902006.

[9] Q.-H. Wei, C. Bechinger, D. Rudhardt, and P. Leiderer, Phys. Rev. Lett. 81, 2606 (1998).

[10] C. Bechinger, M. Brunner, and P. Leiderer, Phys. Rev. Lett. 86, 930 (2001).

[11] E. Frey, D. R. Nelson, and L. Radzihovsky, Phys. Rev. Lett. 83, 2977 (1999).

[12] L. Radzihovsky, E. Frey, and D. R. Nelson, Phys. Rev. E 63, 031503(2001).

[13] W. Strepp, S. Sengupta, and P. Nielaba, Phys. Rev. E 66, 056109 (2002).

[14] W. Strepp, S. Sengupta, and P. Nielaba, Phys. Rev. E 63 046106(2001).

[15] W. Strepp and P. Nielaba (to be published).

[16] M. Brunner, C. Bechinger, W. Strepp, V. Lobaskin, and H. H.V. Grünberg, Europhys. Lett. 58, 926 (2002).

[17] Equation (1) was derived assuming pointlike particles.

[18] G. Nägele, T. Zwick, R. Krause, and R. Klein, J. Colloid Interface Sci. 161, 347 (1993).

[19] H. Löwen, Phys. Rev. E 53, R29 (1996). 